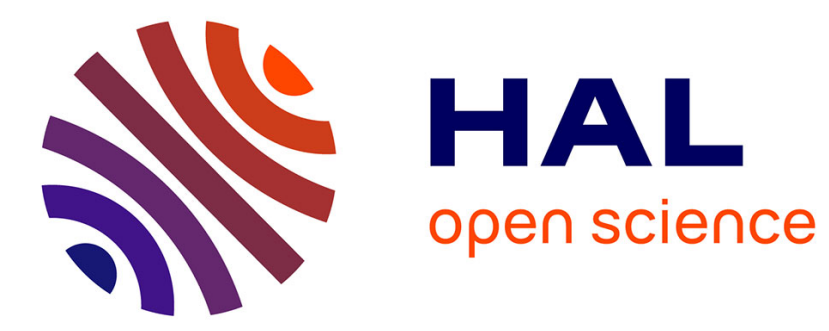

\title{
On some dynamic thermal non clamped contact problems
}

Samir Adly, Oanh Chau

\section{To cite this version:}

Samir Adly, Oanh Chau. On some dynamic thermal non clamped contact problems. Mathematical Programming, Series A, 2013, 139, pp.5-26. 10.1007/s10107-013-0657-9 . hal-00907084

\section{HAL Id: hal-00907084 https://hal.science/hal-00907084}

Submitted on 11 Oct 2018

HAL is a multi-disciplinary open access archive for the deposit and dissemination of scientific research documents, whether they are published or not. The documents may come from teaching and research institutions in France or abroad, or from public or private research centers.
L'archive ouverte pluridisciplinaire HAL, est destinée au dépôt et à la diffusion de documents scientifiques de niveau recherche, publiés ou non, émanant des établissements d'enseignement et de recherche français ou étrangers, des laboratoires publics ou privés. 


\title{
On some dynamic thermal non clamped contact problems
}

\author{
Samir Adly • Oanh Chau
}

\begin{abstract}
We study a class of dynamic thermal sub-differential contact problems with friction, for long memory visco-elastic materials, without the clamped condition, which can be put into a general model of system defined by a second order evolution inequality, coupled with a first order evolution equation. We present and establish an existence and uniqueness result, by using general results on first order evolution inequality, with monotone operators and fixed point methods. Finally a fully discrete scheme for numerical approximations is provided, and corresponding various numerical computations in dimension two will be given.
\end{abstract}

Keywords Long memory thermo-visco-elasticity - Sub-differential contact condition · Non clamped condition · Dynamic process · Fixed point ·

Evolution inequality $\cdot$ Numerical simulations

\section{Introduction}

Because of their impacts in every life, contact mechanics still remain a rich domain of research, and the literature devoted to various aspects of the subject is growing. An early attempt at the study of contact problems for elastic viscoelastic materials within

Dedicated to Jon Borwein in honor of his 60th birthday.

S. Adly ( $\square)$

Laboratoire XLIM, Université de Limoges, 87060 Limoges Cedex, France

e-mail: samir.adly@unilim.fr

O. Chau

Université de La Réunion, 97715 Saint-Denis Messag cedex 9, La Réunion, France

e-mail: oanh.chau@univ-reunion.fr 
the mathematical analysis framework was introduced in the pioneering reference works $[6,7,15,17]$. Further extensions to non convex contact conditions with non-monotone and possible multi-valued constitutive laws led to the active domain of non-smooth mechanic within the framework of the so-called hemivariational inequalities, for a mathematical as well as mechanical treatment we refer to [10] and [18]. Difficult problems with the lack of coerciveness, treated by non-coercive variational inequalities, can be found in [1].

However, multiple contact problems are still open since the years 1970s: such as uniqueness of static Signorini Coulomb frictional problems, solvability of dynamic problems for purely elastic bodies, existence of energy conserving solutions etc., for details see e.g. [7] or [8].

Our intention is not to solve these very hard problems. Here we are dealing with infinitesimal model of thermal frictional problems for viscoelastic bodies. Firstly the contact is governed by a general sub-differential condition on the velocity, which includes several kinds of friction laws, such as Tresca's one, or damped conditions. This type of laws does not verify the model of impenetrability of mass, as in Signorini's condition. However, it is a good approximation in our infinitesimal model. For this reason, it is frequently used in the literature, such as in [14]. Secondly, in our viscoelastic motion, by the thermodynamic law of conservation of energy, the dissipated mechanical energy is transformed into thermal energy. Then we include thermal effects by a heat equation in the viscoelastic system, putting then the problem into a coupled system, defined by a second order evolution inequality, and a differential equation verified by the temperature. Of course, viscosity generates heat, adding in the weak formulation some quadratic form of the linearized deformation, that we neglect in our infinitesimal model. As a complement, more realistic complete models in thermoviscoelastic systems, with other frictional laws as Coulomb's or Signorini's, by taking into account the viscous heat, could be found for example in [8]. On the other hand, infinitesimal frictional models are widely studied in contact literature, see e.g. [14], which remain approximative models, with some limitations on the impenetrability of mass condition, or on the appropriated conservation laws of thermodynamics.

In [14], the authors study the infinitesimal anti-plane shear deformation of a cylindric body, which is subjected to given surface and volume forces, in frictional contact with a rigid foundation. The main purpose in this book is to show the cross-fertilization between various two-dimensional frictional new and nonstandard models arising in the anti-plane shear contact mechanics on the one hand, and numerous types of abstract variational inequalities, on the other hand. Their the motion process is either static or quasistatic. The material constitutive law could be purely elastic, or viscoelastic, of short or long memory type. The frictional contact has been modeled by various versions of slip-dependent or regularized types of Coulomb's or Tresca's power laws. Existence, uniqueness, regularity and convergence results of weak solution on displacement field have been proved.

This work is a continuation of the results obtained in [14]. We extend the previous static or quasistatic anti-plane contact problems into a three dimensional dynamic thermal problem, with the presentation of numerical computations. The important point here is that the usual clamped condition has been deleted. The main difficulty is that Korn's inequality cannot be applied any more. For this proposal, following the 
technic already developed by Duvaut and Lions [7] for Coulomb's friction models, we use the inertial term of the dynamic process to compensate the loss of coerciveness in the a priori estimates, for our general sub-differential contact condition. By change of variable, we bring the coupled second order evolution inequality into a classical first order evolution inequality. Then adopting fixed point methods frequently used in [14], we prove an existence and uniqueness of displacement and temperature fields, using monotonicity and convexity properties. Finally, to complete the novelties mentioned above, we propose a numerical scheme for the approximation of the solution fields, followed by the corresponding numerical computations.

The paper is organized as follows. In Sect. 2 we describe the mechanical problem, specify the assumptions on the data to derive the variational formulation, and then we state our main existence and uniqueness result. In Sect. 3, we give the proof of the claimed result. In Sect. 4, we present several numerical simulations, showing then the evolution of the displacement and temperature fields, as well as of the Von Mises' stress norm.

\section{Statement of the problem}

In this section we study a class of thermal contact problems with sub-differential conditions, for long memory visco-elastic materials. We describe the mechanical problems, list the assumptions on the data and derive the corresponding variational formulations. Then we state an existence and uniqueness result on displacement and temperature fields, which we will prove in the next section.

The physical setting is as follows. A visco-elastic body occupies the domain $\Omega$ with surface $\Gamma$ that is divided into two disjoint measurable parts, $\Gamma_{F}$ and $\Gamma_{c}$. Let $[0, T]$ be the time interval of interest, where $T>0$. We assume that a volume force of density $\boldsymbol{f}_{0}$ acts in $\Omega \times(0, T)$ and that surface tractions of density $\boldsymbol{f}_{F}$ act on $\Gamma_{F} \times(0, T)$. The body may come in contact with an obstacle, the foundation, over the potential contact surface $\Gamma_{c}$. The model of the contact is specified by a general sub-differential boundary condition, where thermal effects may occur in the frictional contact with the basis. We are interested in the dynamic evolution of the body.

Let us recall now some classical notations, see e.g. [7,16] for further details. We denote by $S_{d}$ the space of second order symmetric tensors on $\mathbb{R}^{d}(d=2,3)$, while "." and $|\cdot|$ will represent the inner product and the Euclidean norm on $S_{d}$ and $\mathbb{R}^{d}$. Let $\Omega \subset \mathbb{R}^{d}$ be a bounded domain with a Lipschitz boundary $\Gamma$ and let $\boldsymbol{v}$ denote the unit outer normal on $\Gamma$. Everywhere in the sequel the indices $i$ and $j$ run from 1 to $d$, summation over repeated indices is implied and the index that follows a comma represents the partial derivative with respect to the corresponding component of the independent variable. We also use the following notation:

$$
\begin{aligned}
H & =\left(L^{2}(\Omega)\right)^{d}, \quad \mathcal{H}=\left\{\boldsymbol{\sigma}=\left(\sigma_{i j}\right) \mid \sigma_{i j}=\sigma_{j i} \in L^{2}(\Omega), 1 \leq i, j \leq d\right\}, \\
H_{1} & =\{\boldsymbol{u} \in H \mid \boldsymbol{\varepsilon}(\boldsymbol{u}) \in \mathcal{H}\}, \quad \mathcal{H}_{1}=\{\boldsymbol{\sigma} \in \mathcal{H} \mid \operatorname{Div} \boldsymbol{\sigma} \in H\} .
\end{aligned}
$$

Here $\boldsymbol{\varepsilon}: H_{1} \longrightarrow \mathcal{H}$ and Div $: \mathcal{H}_{1} \longrightarrow H$ are the deformation and the divergence operators, respectively, defined by: 


$$
\boldsymbol{\varepsilon}(\boldsymbol{u})=\left(\varepsilon_{i j}(\boldsymbol{u})\right), \quad \varepsilon_{i j}(\boldsymbol{u})=\frac{1}{2}\left(u_{i, j}+u_{j, i}\right), \quad \operatorname{Div} \boldsymbol{\sigma}=\left(\sigma_{i j, j}\right)
$$

The spaces $H, \mathcal{H}, H_{1}$ and $\mathcal{H}_{1}$ are real Hilbert spaces endowed with the canonical inner products given by:

$$
\begin{aligned}
(\boldsymbol{u}, \boldsymbol{v})_{H} & =\int_{\Omega} u_{i} v_{i} d x, \quad(\boldsymbol{\sigma}, \boldsymbol{\tau})_{\mathcal{H}}=\int_{\Omega} \sigma_{i j} \tau_{i j} d x, \\
(\boldsymbol{u}, \boldsymbol{v})_{H_{1}} & =(\boldsymbol{u}, \boldsymbol{v})_{H}+(\boldsymbol{\varepsilon}(\boldsymbol{u}), \boldsymbol{\varepsilon}(\boldsymbol{v}))_{\mathcal{H}}, \quad(\boldsymbol{\sigma}, \boldsymbol{\tau})_{\mathcal{H}_{1}}=(\boldsymbol{\sigma}, \boldsymbol{\tau})_{\mathcal{H}}+(\operatorname{Div} \boldsymbol{\sigma}, \operatorname{Div} \boldsymbol{\tau})_{H} .
\end{aligned}
$$

We recall that $C$ denotes the class of continuous functions; and $C^{m}, m \in \mathbb{N}^{*}$ the set of $m$ times continuously differentiable functions.

Finally $\mathcal{D}(\Omega)$ denotes the set of infinitely differentiable real functions with compact support in $\Omega$; and $W^{m, p}, m \in \mathbb{N}, 1 \leq p \leq+\infty$ for the classical Sobolev spaces; and

$$
H_{0}^{m}(\Omega):=\left\{w \in W^{m, 2}(\Omega), w=0 \text { on } \Gamma\right\}, \quad m \geq 1 .
$$

To continue, the mechanical problem is then formulated as follows.

Problem $Q$ : Find a displacement field $\boldsymbol{u}: \Omega \times[0, T] \longrightarrow \mathbb{R}^{d}$ and a stress field $\sigma: \Omega \times[0, T] \longrightarrow S_{d}$ and a temperature field $\theta: \Omega \times[0, T] \longrightarrow \mathbb{R}_{+}$such that for a.e. $t \in(0, T)$ :

$$
\begin{aligned}
& \boldsymbol{\sigma}(t)=\mathcal{A} \boldsymbol{\varepsilon}(\dot{\boldsymbol{u}}(t))+\mathcal{G} \boldsymbol{\varepsilon}(\boldsymbol{u}(t))+\int_{0}^{t} \mathcal{B}(t-s) \boldsymbol{\varepsilon}(\boldsymbol{u}(s)) d s-\theta(t) C_{e} \text { in } \Omega \\
& \ddot{\boldsymbol{u}}(t)=\operatorname{Div} \boldsymbol{\sigma}(t)+\boldsymbol{f}_{0}(t) \text { in } \Omega \\
& \boldsymbol{\sigma}(t) v=\boldsymbol{f}_{F}(t) \text { on } \Gamma_{F} \\
& \boldsymbol{u}(t) \in U, \quad \varphi(\boldsymbol{w})-\varphi(\dot{\boldsymbol{u}}(t)) \geq-\boldsymbol{\sigma}(t) \boldsymbol{v} \cdot(\boldsymbol{w}-\dot{\boldsymbol{u}}(t)) \quad \forall \boldsymbol{w} \in U \quad \text { on } \Gamma_{c} \\
& \dot{\theta}(t)-\operatorname{div}\left(K_{c} \nabla \theta(t)\right)=-c_{i j} \frac{\partial \dot{u}_{i}}{\partial x_{j}}(t)+q(t) \text { on } \Omega \\
& -k_{i j} \frac{\partial \theta}{\partial x_{j}}(t) n_{i}=k_{e}\left(\theta(t)-\theta_{R}\right) \text { on } \Gamma_{c} \\
& \theta(t)=0 \quad \text { on } \quad \Gamma_{F} \\
& \theta(0)=\theta_{0} \quad \text { in } \Omega \\
& \boldsymbol{u}(0)=\boldsymbol{u}_{0}, \quad \dot{\boldsymbol{u}}(0)=\boldsymbol{v}_{0} \quad \text { in } \Omega
\end{aligned}
$$

Here, (2.1) is the Kelving Voigt's long memory thermo-visco-elastic constitutive law of the body, $\sigma$ the stress tensor, $\mathcal{A}$ is the viscosity operator, $\mathcal{G}$ for the elastic operator, $C_{e}:=\left(c_{i j}\right)$ represents the thermal expansion tensor, and $\mathcal{B}$ is the so called tensor of relaxation which defines the long memory of the material, as an important particular case, when $\mathcal{B} \equiv 0$, we find again the usual visco-elasticity of short memory. In (2.1) is 
the dynamic equation of motion where the mass density $\varrho \equiv 1$. On the contact surface, the general relation (2.4) is a sub-differential boundary condition, where

$$
\mathcal{D}(\Omega)^{d} \subset U
$$

represents the set of contact admissible test functions, $\sigma \boldsymbol{v}$ denotes the Cauchy stress vector on the contact boundary and $\varphi: \Gamma_{c} \times \mathbb{R}^{d} \longrightarrow \mathbb{R}$ is a given function. Various situations may be modeled by such a condition, and some concrete examples will be recalled below. The differential equation (2.5) describes the evolution of the temperature field, where $K_{c}:=\left(k_{i j}\right)$ represents the thermal conductivity tensor, $q(t)$ the density of volume heat sources. The associated temperature boundary condition is given by (2.6), where $\theta_{R}$ is the temperature of the foundation, and $k_{e}$ is the heat exchange coefficient between the body and the obstacle. Finally, $\boldsymbol{u}_{0}, \boldsymbol{v}_{0}, \theta_{0}$ represent the initial displacement, velocity and temperature, respectively.

One may remark that since $\varphi$ is assumed real-valued, then unilateral contact, defined by indicator functions taking infinite values, is excluded. So the body is in fixed contact with the foundation of the body according to a friction law. This is consistent with the linear heat conduction modeled in (2.5). In the following Example 1 with the Tresca's friction law, as the contact is bilateral, one might consider the problem between two deformable bodies.

We insist that the new feature here is due to the absence of the usual claimed condition. However, there is coerciveness with regard to the temperature by (2.6).

To derive the variational formulation of the mechanical problems (2.1)-(2.9) we need additional notations. Thus, let $V$ denote the closed subspace of $H_{1}$ defined by

$$
\begin{aligned}
& \mathcal{D}(\Omega)^{d} \subset V=H_{1} \cap U ; \\
& E=\left\{\eta \in H^{1}(\Omega), \eta=0 \text { on } \Gamma_{F}\right\} .
\end{aligned}
$$

We remark that the subspace $V$ may be different or not to the whole space $H_{1}$, depending on the set $U$ of admissible contact conditions (see the two following Examples 1 and 2).

On $V$ we consider the inner product given by

$$
(\boldsymbol{u}, \boldsymbol{v})_{V}=(\boldsymbol{\varepsilon}(\boldsymbol{u}), \boldsymbol{\varepsilon}(\boldsymbol{v}))_{\mathcal{H}}+(\boldsymbol{u}, \boldsymbol{v})_{H} \quad \forall \boldsymbol{u}, \boldsymbol{v} \in V,
$$

and let $\|\cdot\|_{V}$ be the associated norm, i.e.

$$
\|\boldsymbol{v}\|_{V}^{2}=\|\boldsymbol{\varepsilon}(\boldsymbol{v})\|_{\mathcal{H}}^{2}+\|\boldsymbol{v}\|_{H}^{2} \quad \forall \boldsymbol{v} \in V
$$

It follows that $\|\cdot\|_{H_{1}}$ and $\|\cdot\|_{V}$ are equivalent norms on $V$ and therefore $\left(V,\|\cdot\|_{V}\right)$ is a real Hilbert space. Moreover, by the Sobolev's trace theorem, we have a constant $C_{0}>0$ depending only on $\Omega$, and $\Gamma_{c}$ such that

$$
\|\boldsymbol{v}\|_{L^{2}\left(\Gamma_{c}\right)} \leq C_{0}\|\boldsymbol{v}\|_{V} \quad \forall \boldsymbol{v} \in V
$$


In the study of the mechanical problem (2.1)-(2.9), we assume that the viscosity operator $\mathcal{A}: \Omega \times S_{d} \longrightarrow S_{d},(\boldsymbol{x}, \boldsymbol{\tau}) \longmapsto\left(a_{i j k h}(\boldsymbol{x}) \tau_{k h}\right)$ is linear on the second variable and satisfies the usual properties of ellipticity and symmetry, i.e.

$$
\left\{\begin{array}{l}
\text { (i) } a_{i j k h} \in W^{1, \infty}(\Omega) ; \\
\text { (ii) } \mathcal{A} \boldsymbol{\sigma} \cdot \boldsymbol{\tau}=\boldsymbol{\sigma} \cdot \mathcal{A} \boldsymbol{\tau} \forall \boldsymbol{\sigma}, \boldsymbol{\tau} \in S_{d}, \text { a.e. in } \Omega \\
\text { (iii) there exists } m_{\mathcal{A}}>0 \text { such that } \\
\mathcal{A} \boldsymbol{\tau} \cdot \boldsymbol{\tau} \geq m_{\mathcal{A}}|\boldsymbol{\tau}|^{2} \quad \forall \boldsymbol{\tau} \in S_{d}, \text { a.e. in } \Omega
\end{array}\right.
$$

The elasticity operator $\mathcal{G}: \Omega \times S_{d} \longrightarrow S_{d}$ satisfies :

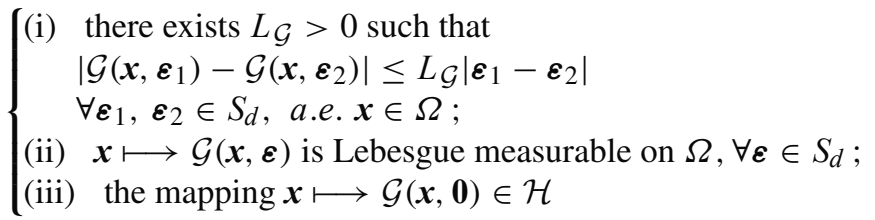

The relaxation tensor $\mathcal{B}:[0, T] \times \Omega \times S_{d} \longrightarrow S_{d},(t, \boldsymbol{x}, \boldsymbol{\tau}) \longmapsto\left(B_{i j k h}(t, \boldsymbol{x}) \tau_{k h}\right)$ satisfies

$$
\left\{\begin{array}{l}
\text { (i) } B_{i j k h} \in W^{1, \infty}\left(0, T ; L^{\infty}(\Omega)\right) \\
\text { (ii) } \mathcal{B}(t) \boldsymbol{\sigma} \cdot \boldsymbol{\tau}=\boldsymbol{\sigma} \cdot \mathcal{B}(t) \boldsymbol{\tau} \\
\quad \forall \boldsymbol{\sigma}, \boldsymbol{\tau} \in S_{d}, \text { a.e. } t \in(0, T) \text {, a.e. in } \Omega
\end{array}\right.
$$

We suppose the body forces and surface tractions satisfy

$$
\boldsymbol{f}_{0} \in W^{1,2}(0, T ; H), \quad \boldsymbol{f}_{F} \in W^{1,2}\left(0, T ; L^{2}\left(\Gamma_{F}\right)^{d}\right)
$$

For the thermal tensors and the heat sources density, we suppose that

$$
C_{e}=\left(c_{i j}\right), \quad c_{i j}=c_{j i} \in L^{\infty}(\Omega), \quad q \in W^{1,2}\left(0, T ; L^{2}(\Omega)\right)
$$

The boundary thermal data satisfy

$$
k_{e} \in L^{\infty}\left(\Omega ; \mathbb{R}^{+}\right), \quad \theta_{R} \in W^{1,2}\left(0, T ; L^{2}\left(\Gamma_{c}\right)\right)
$$

The thermal conductivity tensor verifies the usual symmetry end ellipticity: for some $c_{k}>0$ and for all $\left(\xi_{i}\right) \in \mathbb{R}^{d}$,

$$
K_{c}=\left(k_{i j}\right), \quad k_{i j}=k_{j i} \in L^{\infty}(\Omega), \quad k_{i j} \xi_{i} \xi_{j} \geq c_{k} \xi_{i} \xi_{i}
$$

Finally we have to put technical assumptions on the initial data and the sub-differential condition on the contact surface, in order to use classical results on first order set valued evolution equations. Many various possibilities could be considered (see e.g. $[2,3,12])$. Here we use a general theorem taken in $[12$, p. 46], in a simplified case, which is enough for our proposal and applications. 
We assume that the initial data satisfy the conditions

$$
\boldsymbol{u}_{0} \in V, \quad \boldsymbol{v}_{0} \in V \cap H_{0}^{2}(\Omega)^{d}, \quad \theta_{0} \in E \cap H_{0}^{2}(\Omega)
$$

On the contact surface, the following frictional contact function

$$
\psi(\boldsymbol{w}):=\int_{\Gamma_{c}} \varphi(\boldsymbol{w}) d a
$$

verifies

(i) $\psi: V \longrightarrow \mathbb{R}$ is well defined, continuous and convex;

(ii) there exists a sequence of differentiable convex functions $\left(\psi_{n}\right): V \longrightarrow \mathbb{R}$ such that $\forall \boldsymbol{w} \in L^{2}(0, T ; V)$, $\int_{0}^{T} \psi_{n}(\boldsymbol{w}(t)) d t \longrightarrow \int_{0}^{T} \psi(\boldsymbol{w}(t)) d t, n \longrightarrow+\infty$

(iii) for all sequence $\left(\boldsymbol{w}_{n}\right)$ and $\boldsymbol{w}$ in $W^{1,2}(0, T ; V)$ such that $\boldsymbol{w}_{n} \rightarrow \boldsymbol{w}, \boldsymbol{w}_{n}^{\prime} \rightarrow \boldsymbol{w}^{\prime}$ weakly in $L^{2}(0, T ; V)$, then $\liminf _{n \longrightarrow+\infty} \int_{0}^{T} \psi_{n}\left(\boldsymbol{w}_{n}(t)\right) d t \geq \int_{0}^{T} \psi(\boldsymbol{w}(t)) d t$ (iv) $\forall \boldsymbol{w} \in V, \quad\left(\boldsymbol{w}=0\right.$ on $\left.\Gamma_{c} \Longrightarrow \forall n \in \mathbb{N}, \quad \psi_{n}^{\prime}(\boldsymbol{w})=0_{V^{\prime}}\right)$

Here $\psi_{n}^{\prime}(\boldsymbol{v})$ denotes the Fréchet derivative of $\psi_{n}$ at $\boldsymbol{v}$.

To continue, using Green's formula, we obtain the variational formulation of the mechanical problem $Q$ in abstract form as follows.

Problem $Q V:$ Find $\boldsymbol{u}:[0, T] \rightarrow V, \theta:[0, T] \rightarrow E$ satisfying a.e. $t \in(0, T)$ :

$$
\left\{\begin{array}{l}
\langle\ddot{\boldsymbol{u}}(t)+A \dot{\boldsymbol{u}}(t)+B \boldsymbol{u}(t)+C \theta(t), \boldsymbol{w}-\dot{\boldsymbol{u}}(t)\rangle_{V^{\prime} \times V} \\
\quad+\left(\int_{0}^{t} B(t-s) \boldsymbol{\varepsilon}(\boldsymbol{u}(s)) d s, \boldsymbol{\varepsilon}(\boldsymbol{w})-\boldsymbol{\varepsilon}(\dot{\boldsymbol{u}}(t))\right)_{\mathcal{H}}+\psi(\boldsymbol{w})-\psi(\dot{\boldsymbol{u}}(t)) \\
\quad \geq\langle\boldsymbol{f}(t), \boldsymbol{w}-\dot{\boldsymbol{u}}(t)\rangle_{V^{\prime} \times V} \quad \forall \boldsymbol{w} \in V \\
\dot{\theta}(t)+K \theta(t)=R \dot{\boldsymbol{u}}(t)+Q(t) \quad \text { in } \quad E^{\prime} \\
\boldsymbol{u}(0)=\boldsymbol{u}_{0}, \quad \dot{\boldsymbol{u}}(0)=v_{0}, \quad \theta(0)=\theta_{0}
\end{array}\right.
$$

Here, the operators and functions $A, B: V \longrightarrow V^{\prime}, C: E \longrightarrow V^{\prime}, \psi: V \longrightarrow$ $\mathbb{R}, K: E \longrightarrow E^{\prime}, R: V \longrightarrow E^{\prime}, \boldsymbol{f}:[0, T] \longrightarrow V^{\prime}$, and $Q:[0, T] \longrightarrow E^{\prime}$ are defined by $\forall \boldsymbol{v} \in V, \forall \boldsymbol{w} \in V, \forall \tau \in E, \forall \eta \in E$ :

$$
\begin{aligned}
\langle A \boldsymbol{v}, \boldsymbol{w}\rangle_{V^{\prime} \times V} & =(\mathcal{A}(\boldsymbol{\varepsilon} \boldsymbol{v}), \boldsymbol{\varepsilon} \boldsymbol{w})_{\mathcal{H}} ; \\
\langle B \boldsymbol{v}, \boldsymbol{w}\rangle_{V^{\prime} \times V} & =(\mathcal{G}(\boldsymbol{\varepsilon} \boldsymbol{v}), \boldsymbol{\varepsilon} \boldsymbol{w})_{\mathcal{H}} ; \\
\langle C \tau, \boldsymbol{w}\rangle_{V^{\prime} \times V} & =-\left(\tau C_{e}, \boldsymbol{\varepsilon} \boldsymbol{w}\right)_{\mathcal{H}} ; \\
\langle\boldsymbol{f}(t), \boldsymbol{w}\rangle_{V^{\prime} \times V} & =\left(\boldsymbol{f}_{0}(t), \boldsymbol{w}\right)_{H}+\left(\boldsymbol{f}_{F}(t), \boldsymbol{w}\right)_{\left(L^{2}\left(\Gamma_{F}\right)\right)^{d}} ; \\
\langle Q(t), \eta\rangle_{E^{\prime} \times E} & =\int_{\Gamma_{c}} k_{e} \theta_{R}(t) \eta d x+\int_{\Omega} q(t) \eta d x ;
\end{aligned}
$$




$$
\begin{aligned}
\langle K \tau, \eta\rangle_{E^{\prime} \times E} & =\sum_{i, j=1}^{d} \int_{\Omega} k_{i j} \frac{\partial \tau}{\partial x_{j}} \frac{\partial \eta}{\partial x_{i}} d x+\int_{\Gamma_{c}} k_{e} \tau \cdot \eta d a \\
\langle R \boldsymbol{v}, \eta\rangle_{E^{\prime} \times E} & =-\int_{\Omega} c_{i j} \frac{\partial v_{i}}{\partial x_{j}} \eta d x .
\end{aligned}
$$

Theorem 1 Assume that (2.10)-(2.18) hold, then there exists an unique solution $\{\boldsymbol{u}, \theta\}$ to problem $Q V$ with the regularity:

$$
\left\{\begin{array}{l}
\boldsymbol{u} \in W^{2,2}(0, T ; V) \cap W^{2, \infty}(0, T ; H) \\
\theta \in W^{1,2}(0, T ; E) \cap W^{1, \infty}(0, T ; F)
\end{array}\right.
$$

Before giving the proof, we show in the following two typical examples of subdifferential conditions.

\section{Example 1 Bilateral contact with Tresca's friction law}

The contact condition on $\Gamma_{c}$ is bilateral, and satisfies (see e.g. [7,17]):

$$
\left\{\begin{array}{l}
u_{v}=0, \quad\left|\boldsymbol{\sigma}_{\tau}\right| \leq g, \\
\left|\boldsymbol{\sigma}_{\tau}\right|<g \Longrightarrow \dot{\boldsymbol{u}}_{\tau}=\mathbf{0}, \\
\left|\boldsymbol{\sigma}_{\tau}\right|=g \Longrightarrow \dot{\boldsymbol{u}}_{\tau}=-\lambda \boldsymbol{\sigma}_{\tau}, \text { for some } \lambda \geq 0,
\end{array} \quad \text { on } \Gamma_{c} \times(0, T)\right.
$$

Here $g$ represents the friction bound, i.e., the magnitude of the limiting frictional traction at which slip begins, with $g \in L^{\infty}\left(\Gamma_{c}\right), g \geq 0$ a.e. on $\Gamma_{c}$. We deduce the admissible displacement space:

$$
V:=\left\{\boldsymbol{w} \in H_{1} ; \text { with } w_{v}=0 \text { on } \Gamma_{c}\right\}
$$

and the sub-differential contact function:

$$
\varphi(\boldsymbol{x}, \boldsymbol{y})=g(\boldsymbol{x})\left|\boldsymbol{y}_{\tau(x)}\right| \quad \forall \boldsymbol{x} \in \Gamma_{c}, \boldsymbol{y} \in \mathbb{R}^{d},
$$

where $\boldsymbol{y}_{\tau(x)}:=\boldsymbol{y}-y_{v(x)} \boldsymbol{v}(\boldsymbol{x}), y_{v(x)}:=\boldsymbol{y} \cdot \boldsymbol{v}(\boldsymbol{x})$, with $\boldsymbol{v}(\boldsymbol{x})$ the unit normal at $\boldsymbol{x} \in \Gamma_{c}$. We have then

$$
\psi(v):=\int_{\Gamma_{c}} g\left|\boldsymbol{v}_{\tau}\right| d a, \quad \forall v \in V
$$

is well defined on $V$ with the property: for some $c>0$,

$$
|\psi(\boldsymbol{w})-\psi(\boldsymbol{v})| \leq c\|\boldsymbol{v}-\boldsymbol{w}\|_{L^{2}\left(\Gamma_{c}\right)^{d}}, \quad \forall \boldsymbol{v}, \boldsymbol{w} \in V
$$

Let us show that assumptions in (2.18) are verified. 
Firstly it is clear that $\psi: V \longrightarrow \mathbb{R}$ is convex. By using the continuous embedding from $V$ into $L^{2}\left(\Gamma_{c}\right)^{d}$ and the last inequality, we find that

$$
\psi \text { is Lipschitz continuous on } V \text {. }
$$

This gives (2.18)(i). To approximate the function $\psi$, we use the sequence

$$
\psi_{n}(\boldsymbol{v}):=\int_{\Gamma_{c}} g \sqrt{\left|\boldsymbol{v}_{\tau}\right|^{2}+\frac{1}{n}} d a, \quad \forall \boldsymbol{v} \in V, \forall n \in \mathbb{N}^{*}
$$

We verify that the Frechet derivative of $\psi_{n}$ is given by

$$
\psi_{n}^{\prime}(\boldsymbol{v}) . \boldsymbol{h}=\int_{\Gamma_{c}} g \frac{\left(\boldsymbol{v}_{\tau}, \boldsymbol{h}_{\tau}\right)_{\mathbb{R}^{d}}}{\sqrt{\left|\boldsymbol{v}_{\tau}\right|^{2}+\frac{1}{n}}} d a, \quad \forall \boldsymbol{h} \in V .
$$

Then $\psi_{n}$ is of class $C^{1}$.

From the previous formula, it is clear that the derivative $\psi_{n}^{\prime}(n \geq 1)$ is a monotone operator. Then $\psi_{n}$ is convex for all $n \geq 1$. Another longer way to find this property is to use direct algebraic computations. One may show that for all $\alpha \geq 0, \beta \geq 0$ such that $\alpha+\beta=1$, and for all reals $x$ and $y, n \geq 1$ :

$$
\sqrt{(\alpha x+\beta y)^{2}+\frac{1}{n}} \leq \alpha \sqrt{x^{2}+\frac{1}{n}}+\beta \sqrt{y^{2}+\frac{1}{n}} .
$$

The convergence property in (2.18)(ii) follows from Lebesgue's dominated convergence theorem.

To justify (2.18) (iii) we use the following compact embedding theorem (see e.g. [13])

$$
\begin{aligned}
& \boldsymbol{w}_{n} \rightarrow \boldsymbol{w}, \boldsymbol{w}_{n}^{\prime} \rightarrow \boldsymbol{w}^{\prime} \text { weakly in } L^{2}(0, T ; V) \Longrightarrow \boldsymbol{w}_{n} \longrightarrow \boldsymbol{w} \\
& \text { stronly in } L^{2}\left(0, T ; L^{2}(\Gamma)^{d}\right)
\end{aligned}
$$

and the fact that

$$
\psi_{n}\left(\boldsymbol{w}_{n}\right) \geq \psi\left(\boldsymbol{w}_{n}\right), \quad \forall n \in \mathbb{N}^{*},
$$

and from the continuity of $\psi$ :

$$
\int_{0}^{T} \psi\left(\boldsymbol{w}_{n}\right) \longrightarrow \int_{0}^{T} \psi(\boldsymbol{w}), \quad n \longrightarrow+\infty
$$

Finally (2.18) (iv) is immediate from the Frechet derivative of $\psi_{n}$. 
Example 2 Normal damped response and Tresca's friction law

The normal damped response contact condition with Tresca's friction law (see e.g. [5]) is defined by:

$$
\left\{\begin{array}{l}
-\sigma_{v}=k_{0}\left|\dot{u}_{v}\right|^{r-1} \dot{u}_{v}, \quad\left|\boldsymbol{\sigma}_{\tau}\right| \leq g, \\
\left|\boldsymbol{\sigma}_{\tau}\right|<g \Longrightarrow \dot{\boldsymbol{u}}_{\tau}=0, \\
\left|\boldsymbol{\sigma}_{\tau}\right|=g \Longrightarrow \dot{\boldsymbol{u}}_{\tau}=-\lambda \boldsymbol{\sigma}_{\tau}, \quad \text { for some } \lambda \geq 0,
\end{array} \quad \text { on } \Gamma_{c} \times(0, T)\right.
$$

Here $0<r<1$ and $g, k_{0} \in L^{\infty}\left(\Gamma_{c}\right), g \geq 0, k_{0} \geq 0$. The coefficient $k_{0}$ represents the hardness of the foundation, and $g$ the friction threshold. The admissible displacement space is given by:

$$
V:=H_{1}
$$

and the sub-differential contact function

$$
\varphi(\boldsymbol{x}, \boldsymbol{y})=\frac{1}{r+1} k_{0}(\boldsymbol{x})\left|y_{v(\boldsymbol{x})}\right|^{r+1}+g(\boldsymbol{x})\left|\boldsymbol{y}_{\tau}(\boldsymbol{x})\right| \quad \forall \boldsymbol{x} \in \Gamma_{c}, \boldsymbol{y} \in \mathbb{R}^{d} .
$$

Then denoting by $p:=r+1$, we have the contact function well defined on $V$ by

$$
\psi(\boldsymbol{v}):=\int_{\Gamma_{c}} \frac{k_{0}}{p}\left|v_{v}\right|^{p} d a,+\int_{\Gamma_{c}} g\left|\boldsymbol{v}_{\tau}\right| d a, \quad \forall v \in V .
$$

Here $1<p<2$. Then the mapping $x \geq 0 \longmapsto x^{p}$ is convex, which implies that $\psi$ is convex on $V$. Using the continuous embeddings from $V$ into $L^{2}\left(\Gamma_{c}\right)^{d}$, and from $L^{2}\left(\Gamma_{c}\right)$ into $L^{p}\left(\Gamma_{c}\right)$, we verify also that

$$
\psi \text { is Lipschitz continuous on } V \text {. }
$$

To approximate the function $\psi$ we use the sequence

$$
\psi_{n}(\boldsymbol{v}):=\int_{\Gamma_{c}} \frac{k_{0}}{p}\left(\left|v_{v}\right|^{2}+\frac{1}{n}\right)^{\frac{p}{2}} d a+\int_{\Gamma_{c}} g \sqrt{\left|\boldsymbol{v}_{\tau}\right|^{2}+\frac{1}{n}} d a, \quad \forall \boldsymbol{v} \in V, \forall n \in \mathbb{N}^{*}
$$

We verify that the Frechet derivative of $\psi_{n}$ is given by

$$
\psi_{n}^{\prime}(\boldsymbol{v}) \cdot \boldsymbol{h}:=\int_{\Gamma_{c}} k_{0} \frac{v_{v} h_{\nu}}{\left(\left|v_{v}\right|^{2}+\frac{1}{n}\right)^{1-\frac{p}{2}}} d a+\int_{\Gamma_{c}} g \frac{\left(\boldsymbol{v}_{\tau}, \boldsymbol{h}_{\tau}\right)_{\mathbb{R}^{d}}}{\sqrt{\left|\boldsymbol{v}_{\tau}\right|^{2}+\frac{1}{n}}} d a, \quad \forall \boldsymbol{h} \in V .
$$

From the fact that the mapping $x \geq 0 \longmapsto x^{p}$ is convex increasing, and using (2.20), we verify that $\psi_{n}$ is convex for all $n \geq 1$. Similarly we have the conditions (ii)-(iv) in (2.18) by the same arguments than in the previous example.

Then we conclude that the assumptions in (2.18) are also satisfied in this example. 


\section{Proof of Theorem 1}

The idea is to bring the second order inequality to a first order inequality, using monotone operator, convexity and fixed point arguments, and will be carried out in several steps.

Let us introduce the velocity variable

$$
\boldsymbol{v}=\dot{\boldsymbol{u}}
$$

The system in Problem $Q V$ is then written for a.e. $t \in(0, T)$ :

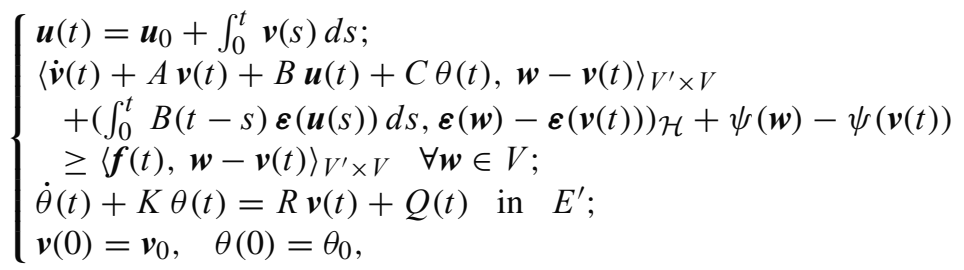

with the regularity

$$
\left\{\begin{array}{l}
\boldsymbol{v} \in W^{1,2}(0, T ; V) \cap W^{1, \infty}(0, T ; H) \\
\theta \in W^{1,2}(0, T ; E) \cap W^{1, \infty}(0, T ; F)
\end{array}\right.
$$

Various abstract formulations concerning the existence and uniqueness result on parabolic variational inequalities of the second kind, could be found in the literature, depending on the assumptions on the operators and data (see e.g. [3,7,12]). We will use a version taken in [7, p. 46], which is sufficient for our proposal, and which we recall as follows.

Theorem 2 Let $A: V \longrightarrow V^{\prime}$ be linear continuous satisfying the following semicoercive condition,

$$
\exists c_{1} \in \mathbb{R}, \exists c_{2}>0, \forall \boldsymbol{w} \in V, \quad\langle A \boldsymbol{w}, \boldsymbol{w}\rangle_{V^{\prime} \times V}+c_{1}\|\boldsymbol{w}\|_{H}^{2} \geq c_{2}\|\boldsymbol{w}\|_{V}^{2}
$$

and $\left\{\psi ;\left(\psi_{n}\right)\right\}$ verifying the hypotheses (2.18), $\mathcal{F} \in W^{1,2}\left(0, T ; V^{\prime}\right)$, and $\boldsymbol{v}_{0} \in V$ satisfying: there exists a sequence $\left(\boldsymbol{v}_{0}^{n}\right)$ in $V$, there exists a bounded sequence $\left(\boldsymbol{h}_{n}\right)$ in $H$ such that $\boldsymbol{v}_{0}^{n} \longrightarrow \boldsymbol{v}_{0}$ in $V$ and for all $n \in \mathbb{N}$,

$$
\left\langle A \boldsymbol{v}_{0}^{n}, \boldsymbol{w}\right\rangle_{V^{\prime} \times V}+\left\langle\psi_{n}^{\prime}\left(\boldsymbol{v}_{0}^{n}\right), \boldsymbol{w}\right\rangle_{V^{\prime} \times V}=\left(\boldsymbol{h}_{n}, \boldsymbol{w}\right)_{H}, \quad \forall \boldsymbol{w} \in V .
$$

Then there exists an unique

$$
\boldsymbol{v} \in W^{1,2}(0, T ; V) \cap W^{1, \infty}(0, T ; H)
$$


satisfying:

$$
\left\{\begin{array}{l}
\langle\dot{\boldsymbol{v}}(t), \boldsymbol{w}-\boldsymbol{v}(t)\rangle_{V^{\prime} \times V}+\langle A \boldsymbol{v}(t), \boldsymbol{w}-\boldsymbol{v}(t)\rangle_{V^{\prime} \times V}+\psi(\boldsymbol{w})-\psi(\boldsymbol{v}(t)) \\
\geq\langle\mathcal{F}(t), \boldsymbol{w}-\boldsymbol{v}(t)\rangle_{V^{\prime} \times V}, \quad \forall \boldsymbol{w} \in V, \quad \text { a.e. } t \in(0, T) \\
\text { and } \boldsymbol{v}(0)=\boldsymbol{v}_{0}
\end{array}\right.
$$

We can apply the Theorem 2: for we have from the definition of $A$ and using (2.10) (iii) that

$$
\forall \boldsymbol{w} \in V, \quad\langle A \boldsymbol{w}, \boldsymbol{w}\rangle_{V^{\prime} \times V} \geq m_{\mathcal{A}}\left(\|\boldsymbol{w}\|_{V}^{2}-\|\boldsymbol{w}\|_{H}^{2}\right)
$$

The conditions on $\boldsymbol{v}_{0}$ in (2.17) imply that there exists a sequence $\left(\boldsymbol{v}_{0}^{n}\right)$ in $\mathcal{D}(\Omega)^{d}$ such that $\boldsymbol{v}_{0}^{n} \longrightarrow \boldsymbol{v}_{0}$ for $\|\cdot\|_{H^{2}(\Omega)^{d}}$. Then

$$
\psi_{n}^{\prime}\left(v_{0}^{n}\right)=0_{V^{\prime}}, \quad \forall n \in \mathbb{N}
$$

and

$$
\boldsymbol{h}_{n}:=A \boldsymbol{v}_{0}^{n}=-\operatorname{Div}\left(\mathcal{A}\left(\boldsymbol{\varepsilon} \boldsymbol{v}_{0}^{n}\right)\right) \longrightarrow-\operatorname{Div}\left(\mathcal{A}\left(\boldsymbol{\varepsilon} \boldsymbol{v}_{0}\right)\right)=A \boldsymbol{v}_{0} \quad \text { in } H .
$$

Thus $\left(\boldsymbol{h}_{n}\right)$ defines a bounded sequence in $H$.

To continue, we assume in the sequel that the conditions (2.10)-(2.18) of the Theorem 1 are satisfied. Let define

$$
\mathcal{W}:=\left\{\eta \in W^{1,2}(0, T ; \mathcal{H}), \eta(0)=\mathcal{G}\left(\boldsymbol{\varepsilon} \boldsymbol{u}_{0}\right)-\theta_{0} C_{e}\right\}
$$

We begin by

Lemma 1 For all $\eta \in \mathcal{W}$, there exists an unique

$$
\boldsymbol{v}_{\eta} \in W^{1,2}(0, T ; V) \cap W^{1, \infty}(0, T ; H)
$$

satisfying

$$
\left\{\begin{array}{l}
\left\langle\dot{\boldsymbol{v}}_{\eta}(t)+A \boldsymbol{v}_{\eta}(t), \boldsymbol{w}-\boldsymbol{v}_{\eta}(t)\right\rangle_{V^{\prime} \times V}+\left(\eta(t), \boldsymbol{\varepsilon}(\boldsymbol{w})-\boldsymbol{\varepsilon}\left(\boldsymbol{v}_{\eta}(t)\right)\right)_{\mathcal{H}} \\
\quad+\psi(\boldsymbol{w})-\psi\left(\boldsymbol{v}_{\eta}(t)\right) \geq\left\langle\boldsymbol{f}(t), \boldsymbol{w}-\boldsymbol{v}_{\eta}(t)\right\rangle_{V^{\prime} \times V} \\
\quad \forall \boldsymbol{w} \in V, \quad \text { a.e. } t \in(0, T) \\
\boldsymbol{v}_{\eta}(0)=\boldsymbol{v}_{0}
\end{array}\right.
$$

Moreover, $\exists c>0$ such that $\forall \eta_{1}, \eta_{2} \in \mathcal{W}$ :

$$
\left\|\boldsymbol{v}_{\eta_{2}}(t)-\boldsymbol{v}_{\eta_{1}}(t)\right\|_{H}^{2}+\int_{0}^{t}\left\|\boldsymbol{v}_{\eta_{1}}-\boldsymbol{v}_{\eta_{2}}\right\|_{V}^{2} \leq c \int_{0}^{t}\left\|\eta_{1}-\eta_{2}\right\|_{\mathcal{H}}^{2}, \quad \forall t \in[0, T] .
$$


Proof Let $\eta \in \mathcal{W}$. The existence and uniqueness of $\boldsymbol{v}_{\eta}$ follows straightly from Theorem 2, where we apply $\mathcal{F}$ defined by for all $t \in[0, T]$,

$$
\langle\mathcal{F}(t), \boldsymbol{w}\rangle_{V^{\prime} \times V}:=\langle f(t), \boldsymbol{w}\rangle_{V^{\prime} \times V}-(\eta(t), \boldsymbol{\varepsilon}(\boldsymbol{w}))_{\mathcal{H}}, \quad \forall \boldsymbol{w} \in V .
$$

The assumptions in (2.13) imply that $\mathcal{F} \in W^{1,2}\left(0, T ; V^{\prime}\right)$.

Now let $\eta_{1}, \eta_{2} \in \mathcal{W}$. In (3.1) we take $\left(\eta=\eta_{1}, \boldsymbol{w}=\boldsymbol{v}_{\eta_{2}}(t)\right)$, then $\left(\eta=\eta_{2}, \boldsymbol{w}=\right.$ $\left.\boldsymbol{v}_{\eta_{1}}(t)\right)$. Adding the two inequalities, we deduce that for a.e. $t \in(0, T)$

$$
\begin{aligned}
& \left\langle\dot{\boldsymbol{v}}_{\eta_{2}}(t)-\dot{\boldsymbol{v}}_{\eta_{1}}(t), \boldsymbol{v}_{\eta_{2}}(t)-\boldsymbol{v}_{\eta_{1}}(t)\right\rangle_{V^{\prime} \times V}+\left\langle A \boldsymbol{v}_{\eta_{2}}(t)-A \boldsymbol{v}_{\eta_{1}}(t), \boldsymbol{v}_{\eta_{2}}(t)-\boldsymbol{v}_{\eta_{1}}(t)\right\rangle_{V^{\prime} \times V} \\
& \quad \leq-\left(\eta_{2}(t)-\eta_{1}(t), \boldsymbol{\varepsilon}\left(\boldsymbol{v}_{\eta_{2}}(t)\right)-\boldsymbol{\varepsilon}\left(\boldsymbol{v}_{\eta_{1}}(t)\right)\right)_{\mathcal{H}} .
\end{aligned}
$$

Then integrating over $(0, t)$, from (2.10) (iii) and from the initial condition on the velocity, we obtain:

$$
\begin{aligned}
\forall t & \in[0, T], \quad\left\|\boldsymbol{v}_{\eta_{2}}(t)-\boldsymbol{v}_{\eta_{1}}(t)\right\|_{H}^{2}+m_{A} \int_{0}^{t}\left\|\boldsymbol{v}_{\eta_{2}}(s)-\boldsymbol{v}_{\eta_{1}}(s)\right\|_{V}^{2} d s \\
\leq & -\int_{0}^{t}\left(\eta_{2}(s)-\eta_{1}(s), \boldsymbol{\varepsilon}\left(\boldsymbol{v}_{\eta_{2}}(s)\right)-\boldsymbol{\varepsilon}\left(\boldsymbol{v}_{\eta_{1}}(s)\right)\right)_{\mathcal{H}} d s+m_{A} \\
& \times \int_{0}^{t}\left\|\boldsymbol{v}_{\eta_{2}}(s)-\boldsymbol{v}_{\eta_{1}}(s)\right\|_{H}^{2} d s .
\end{aligned}
$$

We conclude that $\exists c>0$ such that $\forall \eta_{1}, \eta_{2} \in \mathcal{W}, \forall t \in[0, T]$ :

$$
\begin{aligned}
& \left\|\boldsymbol{v}_{\eta_{2}}(t)-\boldsymbol{v}_{\eta_{1}}(t)\right\|_{H}^{2}+\int_{0}^{t}\left\|\boldsymbol{v}_{\eta_{1}}(s)-\boldsymbol{v}_{\eta_{2}}(s)\right\|_{V}^{2} d s \\
& \leq c \int_{0}^{t}\left\|\eta_{1}(s)-\eta_{2}(s)\right\|_{\mathcal{H}}^{2} d s+c \int_{0}^{t}\left\|\boldsymbol{v}_{\eta_{2}}(s)-\boldsymbol{v}_{\eta_{1}}(s)\right\|_{H}^{2} d s .
\end{aligned}
$$

Now let fix $\tau \in[0, T]$. We have $\forall t \in[0, \tau]$ :

$$
\left\|\boldsymbol{v}_{\eta_{2}}(t)-\boldsymbol{v}_{\eta_{1}}(t)\right\|_{H}^{2} \leq c \int_{0}^{\tau}\left\|\eta_{1}(s)-\eta_{2}(s)\right\|_{\mathcal{H}}^{2}+c \int_{0}^{t}\left\|\boldsymbol{v}_{\eta_{2}}(s)-\boldsymbol{v}_{\eta_{1}}(s)\right\|_{H}^{2} d s .
$$

Using then Gronwall's inequality, we obtain $\forall \tau \in[0, T]$ :

$$
\left\|\boldsymbol{v}_{\eta_{2}}(\tau)-\boldsymbol{v}_{\eta_{1}}(\tau)\right\|_{H}^{2} \leq\left(c \int_{0}^{\tau}\left\|\eta_{1}(s)-\eta_{2}(s)\right\|_{\mathcal{H}}^{2}\right) e^{c T} .
$$


Finally, integrating the last inequality and reporting the result in (3.3), we get (3.2).

Here and below, we denote by $c>0$ a generic constant, which value may change from lines to lines.

Lemma 2 For all $\eta \in \mathcal{W}$, there exists an unique

$$
\theta_{\eta} \in W^{1,2}(0, T ; E) \cap W^{1, \infty}(0, T ; F)
$$

satisfying

$$
\left\{\begin{array}{l}
\dot{\theta}_{\eta}(t)+K \theta_{\eta}(t)=R \boldsymbol{v}_{\eta}(t)+Q(t), \quad \text { in } E^{\prime}, \quad \text { a.e. } t \in(0, T), \\
\theta_{\eta}(0)=\theta_{0} .
\end{array}\right.
$$

Moreover, $\exists c>0$ such that $\forall \eta_{1}, \eta_{2} \in \mathcal{W}$ :

$$
\left\|\theta_{\eta_{1}}(t)-\theta_{\eta_{2}}(t)\right\|_{F}^{2} \leq c \int_{0}^{t}\left\|\boldsymbol{v}_{\eta_{1}}-\boldsymbol{v}_{\eta_{2}}\right\|_{V}^{2}, \quad \forall t \in[0, T] .
$$

and

$$
\left\|\dot{\theta}_{\eta_{1}}(t)-\dot{\theta}_{\eta_{2}}(t)\right\|_{F}^{2} \leq c \int_{0}^{t}\left\|\boldsymbol{v}_{\eta_{1}}-\boldsymbol{v}_{\eta_{2}}\right\|_{V}^{2} \text {, a.e. } t \in(0, T) .
$$

Proof The existence and uniqueness result verifying (3.4) follows from classical result on first order evolution equation, which can be seen as a particular case of Theorem 2 applied to the Gelfand evolution triple (see e.g. [19, p. 416]):

$$
E \subset F \equiv F^{\prime} \subset E^{\prime}
$$

We verify that the operator $K: E \longrightarrow E^{\prime}$ is linear continuous and strongly monotone, and from the expression of the operator $R$,

$$
\boldsymbol{v}_{\eta} \in W^{1,2}(0, T ; V) \Longrightarrow R \boldsymbol{v}_{\eta} \in W^{1,2}(0, T ; F),
$$

as $Q \in W^{1,2}\left(0, T ; E^{\prime}\right)$ then $R \boldsymbol{v}_{\eta}+Q \in W^{1,2}\left(0, T ; E^{\prime}\right)$.

Now for $\eta_{1}, \eta_{2} \in \mathcal{W}$, we have for a.e. $t \in(0 ; T)$ :

$$
\begin{aligned}
& \left\langle\dot{\theta}_{\eta_{1}}(t)-\dot{\theta}_{\eta_{2}}(t), \theta_{\eta_{1}}(t)-\theta_{\eta_{2}}(t)\right\rangle_{E^{\prime} \times E}+\left\langle K \theta_{\eta_{1}}(t)-K \theta_{\eta_{2}}(t), \theta_{\eta_{1}}(t)-\theta_{\eta_{2}}(t)\right\rangle_{E^{\prime} \times E} \\
& \quad=\left\langle R \boldsymbol{v}_{\eta_{1}}(t)-R \boldsymbol{v}_{\eta_{2}}(t), \theta_{\eta_{1}}(t)-\theta_{\eta_{2}}(t)\right\rangle_{E^{\prime} \times E} .
\end{aligned}
$$

Then integrating the last property over $(0, t)$, using the strong monotonicity of $K$ and the Lipschitz continuity of $R: V \longrightarrow E^{\prime}$, we deduce (3.5). 
To continue, from

$$
\dot{\theta}_{\eta} \in L^{2}(0, T ; E)
$$

and from (3.4) which implies

$$
\ddot{\theta}_{\eta}(t)+K \dot{\theta}_{\eta}(t)=R \boldsymbol{u}_{\eta}(t)+\dot{Q}(t) \text { a.e. } t \in(0, T) \Longrightarrow \ddot{\theta}_{\eta} \in L^{2}\left(0, T ; E^{\prime}\right) \text {, }
$$

we deduce that

$$
\dot{\theta}_{\eta} \in C([0, T] ; F) .
$$

Then that for $\eta_{1}, \eta_{2} \in \mathcal{W}$, we have for a.e. $t \in(0 ; T)$ :

$$
\begin{aligned}
& \left\langle\ddot{\theta}_{\eta_{1}}(t)-\ddot{\theta}_{\eta_{2}}(t), \dot{\theta}_{\eta_{1}}(t)-\dot{\theta}_{\eta_{2}}(t)\right\rangle_{E^{\prime} \times E}+\left\langle K \dot{\theta}_{\eta_{1}}(t)-K \dot{\theta}_{\eta_{2}}(t), \dot{\theta}_{\eta_{1}}(t)-\dot{\theta}_{\eta_{2}}(t)\right\rangle_{E^{\prime} \times E} \\
& \quad=\left\langle R \boldsymbol{u}_{\eta_{1}}(t)-R \boldsymbol{u}_{\eta_{2}}(t), \dot{\theta}_{\eta_{1}}(t)-\dot{\theta}_{\eta_{2}}(t)\right\rangle_{E^{\prime} \times E} .
\end{aligned}
$$

Integrating the last property over $(0, t)$, and with similar arguments we deduce

$$
\left\|\dot{\theta}_{\eta_{1}}(t)-\dot{\theta}_{\eta_{2}}(t)\right\|_{F}^{2} \leq c \int_{0}^{t}\left\|\boldsymbol{u}_{\eta_{1}}-\boldsymbol{u}_{\eta_{2}}\right\|_{V}^{2}, \quad \text { a.e. } t \in(0, T) .
$$

And (3.6) follows.

Proof of Theorem 1 We have now all the ingredients to prove the Theorem 1.

Consider the operator $\Lambda: \mathcal{W} \rightarrow \mathcal{W}$ defined by for all $\eta \in \mathcal{W}:$

$$
\Lambda \eta(t)=\mathcal{G}\left(\boldsymbol{\varepsilon}\left(\boldsymbol{u}_{\eta}(t)\right)\right)+\int_{0}^{t} B(t-s) \boldsymbol{\varepsilon}\left(\boldsymbol{u}_{\eta}(s)\right) d s-\theta_{\eta}(t) C_{e}, \quad \forall t \in[0, T],
$$

where

$$
\boldsymbol{u}_{\eta}(t)=\boldsymbol{u}_{0}+\int_{0}^{t} \boldsymbol{v}_{\eta}(s) d s, \forall t \in[0, T] ; \quad \boldsymbol{u}_{\eta} \in W^{2,2}(0, T ; V) .
$$

Then from (2.11), (2.12), and Lemma 2, we deduce that for all $\eta_{1}, \eta_{2} \in \mathcal{W}$, for all $t \in[0, T]:$

$$
\begin{aligned}
\left\|\Lambda \eta_{1}(t)-\Lambda \eta_{2}(t)\right\|_{\mathcal{H}}^{2} & \leq c\left\|\theta_{\eta_{1}}(t)-\theta_{\eta_{2}}(t)\right\|_{F}^{2}+c \int_{0}^{t}\left\|\boldsymbol{v}_{\eta_{1}}(s)-\boldsymbol{v}_{\eta_{2}}(s)\right\|_{V}^{2} d s \\
& \leq c \int_{0}^{t}\left\|\boldsymbol{v}_{\eta_{1}}(s)-\boldsymbol{v}_{\eta_{2}}(s)\right\|_{V}^{2} d s
\end{aligned}
$$


Again from (2.12) and (2.11), we have

$$
\begin{aligned}
& \left\|\frac{d}{d t}\left(\int_{0}^{t} B(t-s) \boldsymbol{\varepsilon}\left(\boldsymbol{u}_{\eta_{1}}(s)\right) d s-\int_{0}^{t} B(t-s) \boldsymbol{\varepsilon}\left(\boldsymbol{u}_{\eta_{2}}(s)\right) d s\right)\right\|_{\mathcal{H}}^{2} \\
& \leq c\left\|\boldsymbol{u}_{\eta_{1}}(t)-\boldsymbol{u}_{\eta_{2}}(t)\right\|_{V}^{2}+c \int_{0}^{t}\left\|\boldsymbol{u}_{\eta_{1}}(s)-\boldsymbol{u}_{\eta_{2}}(s)\right\|_{V}^{2} d s \\
& \leq c \int_{0}^{t}\left\|\boldsymbol{v}_{\eta_{1}}(s)-\boldsymbol{v}_{\eta_{2}}(s)\right\|_{V}^{2} d s .
\end{aligned}
$$

and

$$
\left\|\frac{d}{d t}\left(\mathcal{G}\left(\boldsymbol{\varepsilon}\left(\boldsymbol{u}_{\eta_{1}}(t)\right)\right)-\mathcal{G}\left(\boldsymbol{\varepsilon}\left(\boldsymbol{u}_{\eta_{2}}(t)\right)\right)\right)\right\|_{\mathcal{H}}^{2} \leq c\left\|\boldsymbol{v}_{\eta_{1}}(t)-\boldsymbol{v}_{\eta_{2}}(t)\right\|_{V}^{2}
$$

Then

$$
\left\|\frac{d}{d t}\left(\Lambda \eta_{1}(t)-\Lambda \eta_{2}(t)\right)\right\|_{\mathcal{H}}^{2} \leq c\left\|\boldsymbol{v}_{\eta_{1}}(t)-\boldsymbol{v}_{\eta_{2}}(t)\right\|_{V}^{2}+c \int_{0}^{t}\left\|\boldsymbol{v}_{\eta_{1}}(s)-\boldsymbol{v}_{\eta_{2}}(s)\right\|_{V}^{2} d s
$$

Now using (3.7) and (3.8), after some algebraic manipulations, we have for any $\beta>0$ :

$$
\begin{aligned}
& \int_{0}^{t} e^{-\beta \tau}\left(\left\|\Lambda \eta_{1}(\tau)-\Lambda \eta_{2}(\tau)\right\|_{\mathcal{H}}^{2}+\left\|\dot{\Lambda} \dot{\eta}_{1}(\tau)-\dot{\Lambda} \eta_{2}(\tau)\right\|_{\mathcal{H}}^{2}\right) d \tau \\
& \quad \leq \frac{c}{\beta} \int_{0}^{t} e^{-\beta \tau}\left(\left\|\eta_{1}(\tau)-\eta_{2}(\tau)\right\|_{\mathcal{H}}^{2}+\left\|\dot{\eta}_{1}(\tau)-\dot{\eta}_{2}(\tau)\right\|_{\mathcal{H}}^{2}\right) d \tau .
\end{aligned}
$$

We conclude from the last inequality by contracting principle that the operator $\Lambda$ has a unique fixed point $\eta^{*} \in \mathcal{W}$. We verify then that the functions

$$
\boldsymbol{u}(t):=\boldsymbol{u}_{0}+\int_{0}^{t} \boldsymbol{v}_{\eta^{*}}, \forall t \in[0, T], \quad \theta:=\theta_{\eta^{*}}
$$

are solutions to problem $Q V$ with the regularity (2.19), the uniqueness follows from the uniqueness in Lemmas 1 and 2. 


\section{Numerical computations}

Here we propose a discrete scheme for the approximation of the solution fields on velocity and temperature, and provide the corresponding numerical simulations.

For the discretization methods on evolution inequalities, we refer to [4] or [9] for the general functional analysis, and we recommend [11] in the framework of viscoelastic contact problems.

Now let us consider $\Omega \subset \mathbb{R}^{2}$, a polygonal domain. Let $\mathcal{T}^{h}$ be a regular finite element partition of $\Omega$. Let $V^{h} \subset V$ and $E^{h} \subset E$ be the finite element space consisting of piecewise affine functions, with $h>0$ a discretization parameter. Denote by $\Pi_{V}^{h}: H^{2}(\Omega)^{2} \rightarrow V^{h}$ and $\Pi_{E}^{h}: H^{2}(\Omega) \rightarrow E^{h}$ the corresponding finite element interpolation operator. We divide the time interval $[0, T]$ into $N$ equal parts: $t_{n}=n k, n=0,1, \ldots, N$, with the time step $k=T / N$. For a continuous function $\boldsymbol{w} \in C([0, T] ; X)$ with values in a space $X$, we use the notation $\boldsymbol{w}_{n}=\boldsymbol{w}\left(t_{n}\right) \in X$. Then we introduce the following fully discrete scheme.

Problem $P^{h k}$. Find $\boldsymbol{v}^{h k}=\left\{\boldsymbol{v}_{n}^{h k}\right\}_{n=0}^{N} \subset V^{h}, \theta^{h k}=\left\{\theta_{n}^{h k}\right\}_{n=0}^{N} \subset E^{h}$ such that

$$
\boldsymbol{v}_{0}^{h k}=v_{0}^{h}, \quad \theta_{0}^{h k}=\theta_{0}^{h}
$$

and for $n=1, \cdots, N$,

$$
\begin{aligned}
& \left(\frac{\left.\boldsymbol{v}_{n}^{h k}-\boldsymbol{v}_{n-1}^{h k}, \boldsymbol{w}^{h}-\boldsymbol{v}_{n}^{h k}\right)_{H}+\left\langle A \boldsymbol{v}_{n}^{h k}, \boldsymbol{w}^{h}-\boldsymbol{v}_{n}^{h k}\right\rangle_{V^{\prime} \times V}+\left\langle B \boldsymbol{u}_{n-1}^{h k}, \boldsymbol{w}^{h}-\boldsymbol{v}_{n}^{h k}\right\rangle_{V^{\prime} \times V}}{k}+\psi\left(\boldsymbol{v}_{n}^{h k}\right)\right. \\
& \quad+\left\langle C \theta_{n-1}^{h k}, \boldsymbol{w}^{h}-\boldsymbol{v}_{n}^{h k}\right\rangle_{V^{\prime} \times V}+\psi\left(\boldsymbol{w}^{h}\right)-\psi\left(k \sum_{m=0}^{n-1} \mathcal{B}\left(t_{n}-t_{m}\right) \boldsymbol{\varepsilon}\left(\boldsymbol{u}_{m}^{h k}\right), \boldsymbol{\varepsilon}\left(\boldsymbol{w}^{h}\right)-\boldsymbol{\varepsilon}\left(\boldsymbol{v}_{n}^{h k}\right)\right)_{\mathcal{H}} \\
& \quad+\left(\boldsymbol{f}_{n}, \boldsymbol{w}^{h}-\boldsymbol{v}_{n}^{h k}\right\rangle_{V^{\prime} \times V}, \quad \forall \boldsymbol{w}^{h} \in V^{h} ; \\
& \left(\frac{\theta_{n}^{h k}-\theta_{n-1}^{h k}}{k}, \eta^{h}\right)_{F}+\left\langle K \theta_{n}^{h k}, \eta^{h}\right\rangle_{E^{\prime} \times E} \\
& \quad=\left\langle R \boldsymbol{v}_{n}^{h k}, \eta^{h}\right\rangle_{E^{\prime} \times E}+\left\langle Q_{n}, \eta^{h}\right\rangle_{E^{\prime} \times E}, \quad \forall \eta^{h} \in E^{h} ;
\end{aligned}
$$

where

$$
\boldsymbol{u}_{n}^{h k}=\boldsymbol{u}_{n-1}^{h k}+k \boldsymbol{v}_{n}^{h k}, \quad \boldsymbol{u}_{0}^{h k}=\boldsymbol{u}_{0}^{h}
$$

Here $\boldsymbol{u}_{0}^{h} \in V^{h}, \boldsymbol{v}_{0}^{h} \in V^{h}, \theta_{0}^{h} \in E^{h}$ are approximations of the initial values $\boldsymbol{u}_{0}, \boldsymbol{v}_{0}, \theta_{0}$, defined by

$$
\boldsymbol{u}_{0}^{h}=\Pi_{V}^{h} \boldsymbol{u}_{0}, \quad \boldsymbol{v}_{0}^{h}=\Pi_{V}^{h} \boldsymbol{v}_{0}, \quad \theta_{0}^{h}=\Pi_{E}^{h} \theta_{0} .
$$

Here we provide numerical simulations derived from the previous discrete schemes, by using Matlab computation codes, in the cases of two typical examples of 
Fig. 1 Initial configuration

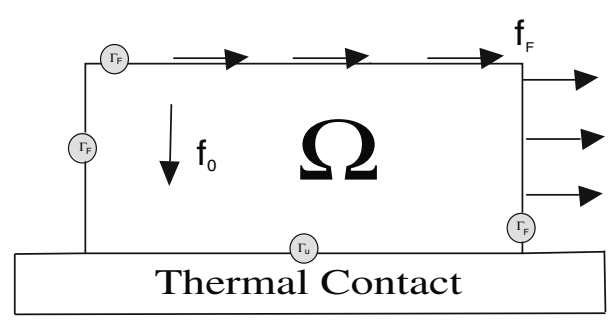

sub-differential conditions, the bilateral contact, and the normal damped response both with Tresca's friction law.

\subsection{Bilateral contact with Tresca's friction law}

We consider for computations a rectangular open set, linear elastic and long memory viscoelastic operators.

$$
\begin{aligned}
& \Omega=\left(0, L_{1}\right) \times\left(0, L_{2}\right) \\
& \Gamma_{F}=\left(\{0\} \times\left[0, L_{2}\right]\right) \cup\left(\left[0, L_{1}\right] \times\left\{L_{2}\right\}\right) \cup\left(\left\{L_{1}\right\} \times\left[0, L_{2}\right]\right) ; \quad \Gamma_{u}=\left[0, L_{1}\right] \times\{0\} ; \\
& (\mathcal{G} \tau)_{i j}=\frac{E \kappa}{1-\kappa^{2}}\left(\tau_{11}+\tau_{22}\right) \delta_{i j}+\frac{E}{1+\kappa} \tau_{i j}, \quad 1 \leq i, j \leq 2, \boldsymbol{\tau} \in S_{2} \\
& (\mathcal{A} \tau)_{i j}=\mu\left(\tau_{11}+\tau_{22}\right) \delta_{i j}+\eta \tau_{i j}, \quad 1 \leq i, j \leq 2, \boldsymbol{\tau} \in S_{2} ; \\
& (\mathcal{B}(t) \tau)_{i j}=B_{1}(t)\left(\tau_{11}+\tau_{22}\right) \delta_{i j}+B_{2}(t) \tau_{i j}, \quad 1 \leq i, j \leq 2, \boldsymbol{\tau} \in S_{2}, t \in[0, T]
\end{aligned}
$$

Here $E$ is the Young's modulus, $\kappa$ the Poisson's ratio of the material and $\delta_{i j}$ denotes the Kronecker symbol, and $\mu$ and $\eta$ are viscosity constants.

We refer to the previous numerical scheme, and use spaces of continuous piecewise affine functions $V^{h} \subset V$ and $E^{h} \subset E$ as families of approximating subspaces. For computations we considered the following data (IS unity), $\forall t \in[0, T]$ :

$$
\begin{aligned}
& L_{1}=L_{2}=1, \quad T=1 \\
& \mu=10, \quad \eta=10, \quad E=2, \quad \kappa=0.1, \quad \boldsymbol{f}_{0}(\boldsymbol{x}, t)=(0,-t) \\
& \boldsymbol{f}_{F}(\boldsymbol{x}, t)=(0,0), \quad \forall \boldsymbol{x} \in\{0\} \times\left[0, L_{2}\right] \\
& \boldsymbol{f}_{F}(\boldsymbol{x}, t)=(1,0), \quad \forall \boldsymbol{x} \in\left(\left[0, L_{1}\right] \times\left\{L_{2}\right\}\right) \cup\left(\left\{L_{1}\right\} \times\left[0, L_{2}\right]\right) \\
& c_{i j}=k_{i j}=k_{e}=1, \quad 1 \leq i, j \leq 2, \quad q=1 \\
& B_{1}(t)=B_{2}(t)=10^{-2} e^{-t} \\
& \boldsymbol{u}_{0}=(0,0), \quad \boldsymbol{v}_{0}=(0,0), \quad \theta_{0}=0
\end{aligned}
$$

In Fig. 1 is representing the initial configuration.

In Fig. 2 the deformed configuration at final time, in the case of visco-elasticity of long memory, where the relaxation coefficients are positive decreasing, for two different types of Tresca's friction bounds. For small friction bound, where $g(x, 0)=$ $\frac{x}{2}, 0 \leq x \leq 1$, we observe on the contact surface a slip phenomena in the direction 

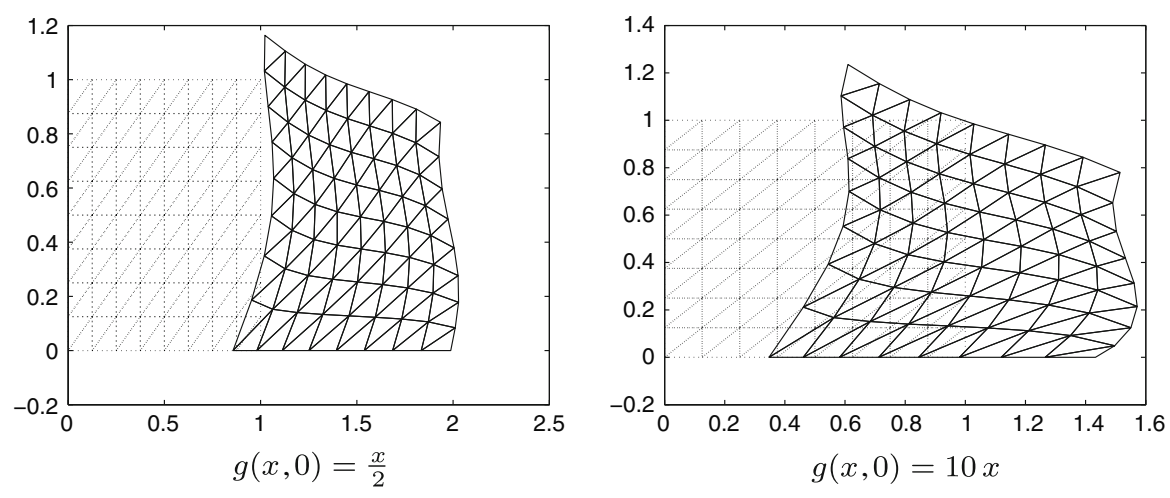

Fig. 2 Long memory deformed configurations at final time, $\theta_{R}(t)=10,0 \leq t \leq 1$
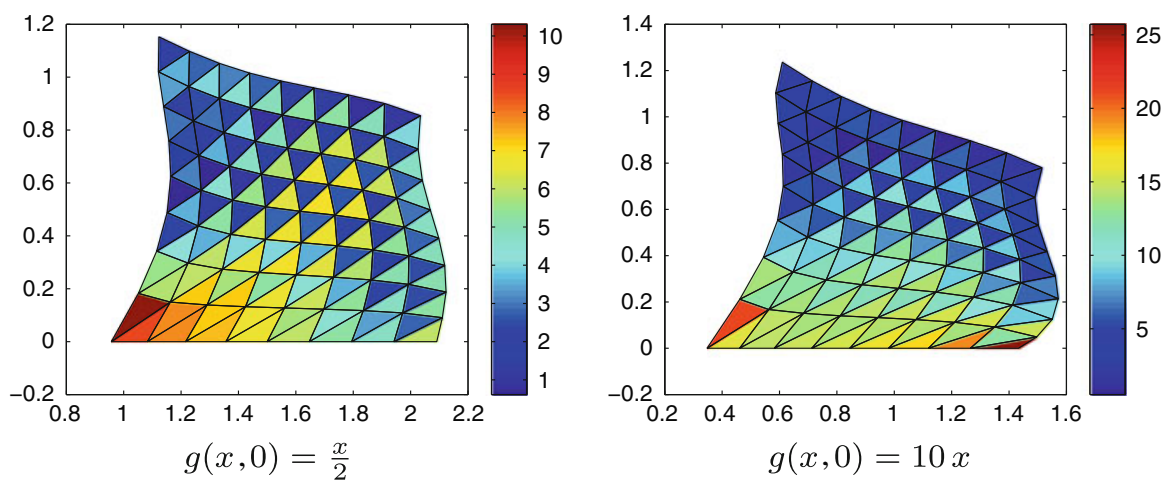

Fig. 3 Von Mises' norm in long memory deformed configurations, $\theta_{R}(t)=10,0 \leq t \leq 1$

of the surface fraction on $\Gamma_{F}$ : which means that the friction bound has been obtained, in the zone of the values of $x$ near to 1 . Whereas for large friction bound, e.g. for $g(x, 0)=10 x, 0 \leq x \leq 1$, then slip in the direction of the traction could not be realized.

In Fig. 3, we compute the Von Mises' norm, for long memory, which gives a global measure of the stress in the body. The maxima of the norm could be seen in the neighborhood of the point $(0,1)$ for small friction bounds, and in the neighborhood of the point $(1,0)$ for large friction bounds.

In Fig. 4, we show the influence of the different temperatures of the foundation $\left(\theta_{R}(t)=0\right.$ or $\left.\theta_{R}(t)=10\right)$ on the temperature field of the body.

\subsection{Normal damped response with Tresca’s friction law}

We take again the rectangular open set, with linear elasticity and visco-elasticity. For computations we used the following data (IS unity), $\forall t \in[0, T]$ : 

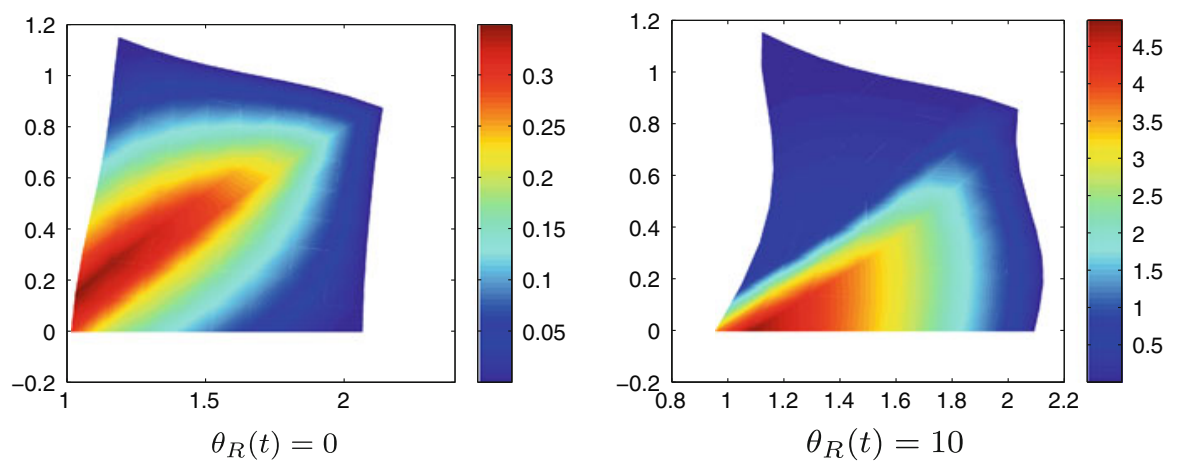

Fig. 4 Temperature field at final time in long memory, $g(x, 0)=\frac{x}{2}, 0 \leq x \leq 1$
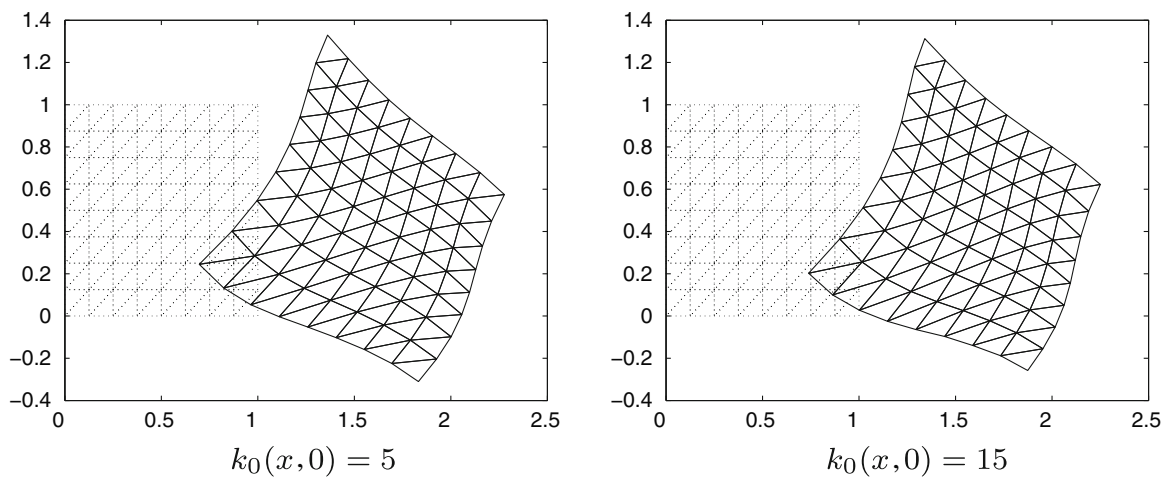

Fig. 5 Long memory deformed configurations at final time, $\theta_{R}(t)=10,0 \leq t \leq 1$
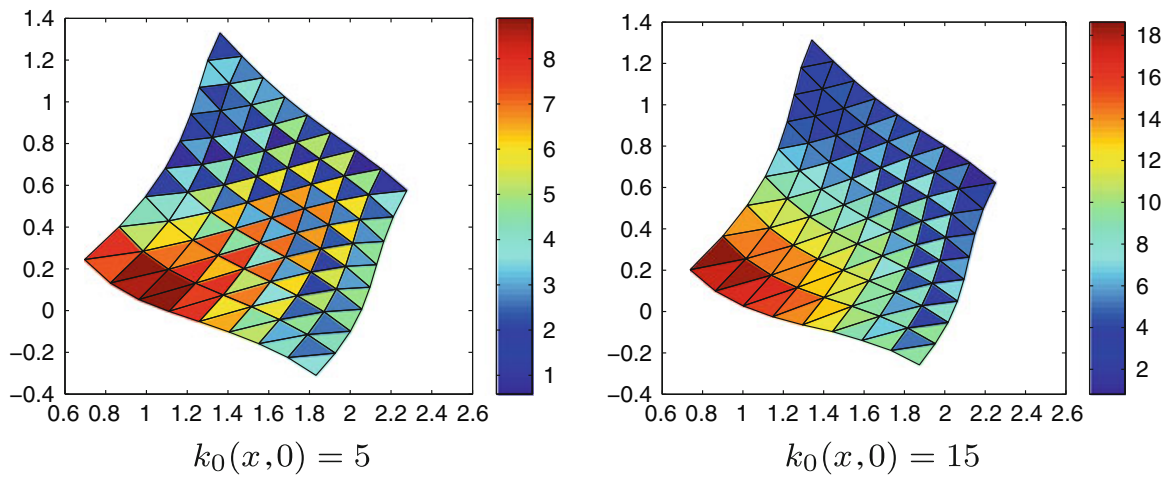

Fig. 6 Von Mises' norm in long memory deformed configurations, $\theta_{R}(t)=10,0 \leq t \leq 1$

$$
\begin{aligned}
& L_{1}=L_{2}=1, \quad T=1 \\
& \mu=10, \quad \eta=10, \quad E=2, \quad \kappa=0.1, \quad \boldsymbol{f}_{0}(\boldsymbol{x}, t)=(0,-t) \\
& \boldsymbol{f}_{F}(\boldsymbol{x}, t)=(0,0), \quad \forall \boldsymbol{x} \in\{0\} \times\left[0, L_{2}\right] \\
& \boldsymbol{f}_{F}(\boldsymbol{x}, t)=(1,0), \quad \forall \boldsymbol{x} \in\left(\left[0, L_{1}\right] \times\left\{L_{2}\right\}\right) \cup\left(\left\{L_{1}\right\} \times\left[0, L_{2}\right]\right)
\end{aligned}
$$



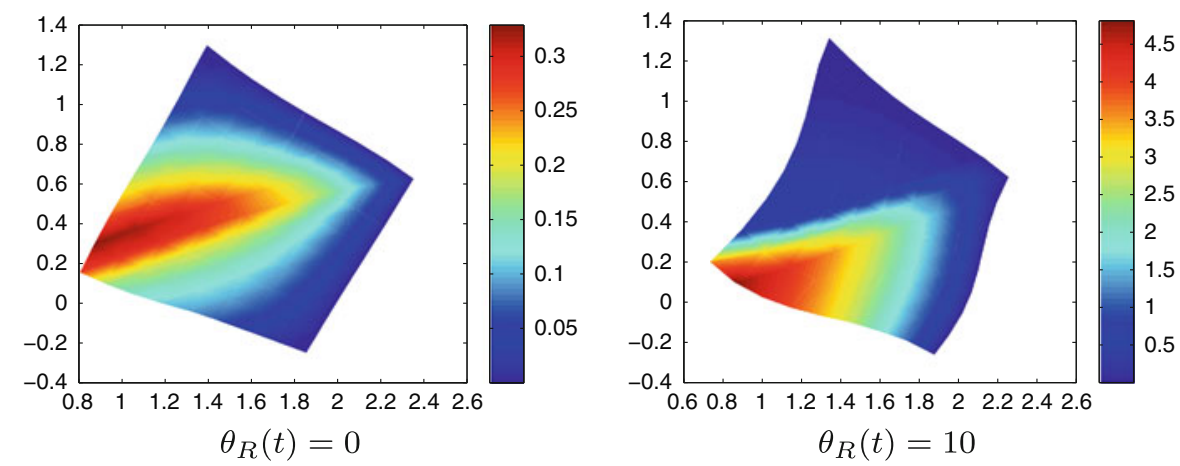

Fig. 7 Temperature field at final time in long memory, $k_{0}(x, 0)=15,0 \leq x \leq 1$

$$
\begin{aligned}
& c_{i j}=k_{i j}=k_{e}=1, \quad 1 \leq i, j \leq 2, \quad q=1 \\
& g(x, 0)=\frac{x}{2}, 0 \leq x \leq 1, \quad r=0.5 \\
& B_{1}(t)=B_{2}(t)=10^{-2} e^{-t} \\
& \boldsymbol{u}_{0}=(0,0), \quad v_{0}=(0,0), \quad \theta_{0}=0
\end{aligned}
$$

We show in Fig. 5 the deformed configuration at final time, through the body for different normal damped response coefficients $k_{0}$, we verify that the penetrability of the foundation depends on its coefficient of hardness. In Fig. 6 we compute the Von Mises' norm, larger stress near the contact surface is then observed for hard obstacle. Finally in Fig. 7 we find again the influence of the temperature of the foundation on the temperature field of the body, and on the final deformed configurations.

Acknowledgments The authors would like to thanks the anonymous referees for many helpful comments and valuable suggestions.

\section{References}

1. Adly, S., Ernst, E., Thera, M.: Stability of the solution set of non-coercive variational inequalities. Commun. Contemp. Math. 4, 145-160 (2002)

2. Barbu, V.: Nonlinear Semigroups and Differential Equations in Banach Spaces. Editura Academiei, Bucharest-Noordhoff, Leyden (1976)

3. Brézis, H.: Problèmes unilatéraux. J. Math. Pures et Appli. 51, 1-168 (1972)

4. Carstensen, C., Gwinner, J.: A theory of discretization for nonlinear evolution inequalities applied to parabolic Signorini problems. Ann. Mat. Pura Appl. 177, 363-394 (1999)

5. Chau, O.: Analyse variationnelle et numérique en mécanique du contact. Thesis, Perpignan, June 2000

6. Ciarlet, P.G.: Mathematical Elasticity, vol. I: Three-Dimensional Elasticity. North-Holland, Amsterdam (1988)

7. Duvaut, G., Lions, J.L.: Les Inéquations en Mécanique et en Physique. Dunod, Paris (1972)

8. Eck, Ch., Jarusek, J., Krbec, M.: Unilateral Contact Problems, Variational Methods and Existence Theorems. Monographs \& Texbooks in Pure \& Applied Mathematics, vol. 270, Chapman and Hall, London (2005)

9. Glowinski, R.: Numerical Methods for Nonlinear Variational Problems. Springer, Berlin (1984) 
10. Goeleven, D., Motreanu, D., Dumont, Y., Rochdi, M.: Variational and Hemivariational Inequalities, Theory, Methods and Applications, Volume I: Unilateral Analysis and Unilateral Mechanics. Kluwer, Dordrecht (2003)

11. Han, W., Sofonea, M.: Evolutionary variational inequalities arising in viscoelastic contact problems. SIAM J. Numer. Anal. 38, 556-579 (2000)

12. Lions, J.L.: Quelques méthodes de résolution des problèmes aux limites non linéaires. Dunod et Gauthier-Villars, Paris (1969)

13. Lions, J.L., Magenes, E.: Problèmes aux limites non homogènes et applications, vol. 1. Dunod, Paris (1968)

14. Matei, A., Sofonea, M.: Variational Inequalities with Applications: A Study of Antiplane Frictional Contact Problems, Advances in Mechanics and Mathematics, vol. 18. Springer, Berlin (2009)

15. Kikuchi, N., Oden, J.T.: Contact Problems in Elasticity. SIAM, Philadelphia (1988)

16. Nečas, J., Hlaváček, I.: Mathematical Theory of Elastic and Elastoplastic Bodies: An Introduction. Elsevier, Amsterdam (1981)

17. Panagiotopoulos, P.D.: Inequality Problems in Meechanics and Applications. Birkhäuser, Basel (1985)

18. Panagiotopoulos, P.D.: Hemivariational Inequalities, Applications in Mechanics and Engineering. Springer, Berlin (1993)

19. Zeidler, E.: Nonlinear Functional Analysis and Its Applications, II/A, Linear Monotone Operators. Springer, Berlin (1997) 